(Se) former pour enseigner le français à ceux qui ne le parlent pas nativement -

\title{
Sur quatre moments de la formation en France des enseignants de français aux étrangers
}

Daniel Coste

\section{(2) OpenEdition Journals}

Édition électronique

URL : https://journals.openedition.org/dhfles/4328

DOI : $10.4000 /$ dhfles.4328

ISSN : 2221-4038

Éditeur

Société Internationale pour l'Histoire du Français Langue Étrangère ou Seconde

Édition imprimée

Date de publication : 1 décembre 2015

Pagination : 171-192

ISSN : 0992-7654

Référence électronique

Daniel Coste, «Sur quatre moments de la formation en France des enseignants de français aux étrangers », Documents pour l'histoire du français langue étrangère ou seconde [En ligne], 55 | 2015, mis en ligne le 01 janvier 2018, consulté le 26 mars 2023. URL : http://journals.openedition.org/dhfles/ 4328 ; DOI : https://doi.org/10.4000/dhfles.4328

Ce document a été généré automatiquement le 26 mars 2023.

Tous droits réservés 


\title{
Sur quatre moments de la formation en France des enseignants de français aux étrangers
}

\author{
Daniel Coste
}

1 La postface, plus encore que la préface, n'a rien d'un genre suffisamment défini et codifié pour poser des contraintes fortes à qui s'y aventure. On peut même s'autoriser à sortir du cadre strict de la thématique ou de la période dont traite l'ouvrage ou le numéro d'une revue, voire se permettre de ne référer directement à aucun des contributeurs au volume. En la circonstance, les considérations qui suivent ni ne reposent toutes sur un travail d'archives ni ne se limitent au siècle sur lequel se concentrent les articles ici rassemblés. Pour autant, elles tendent, dans leur généralité comme dans les cas examinés, à mettre en évidence des facteurs entrant en jeu de manière transversale (synchronique comme diachronique) dans le développement institutionnel de la formation des enseignants de langues vivantes.

2 Sous cette optique, il y a lieu de faire jouer différentes dimensions qui, même si elles ne sont pas toutes toujours présentes simultanément, accompagnent ce développement et sont de nature à fournir une grille de lecture pour l'analyse de moments particuliers. D'entre ces dimensions, la professionnalisation est évidemment une composante majeure, mais non la seule; elle se décline dans un rapport à d'autres facteurs. Il convient aussi de ne pas négliger les conjonctions plus ou moins favorables qui résultent de circonstances historiques données et de ne pas oublier les rôles d'acteurs individuels ou collectifs à même de tirer parti de telles conjonctions.

Dans ce qui suit, ce sont quatre moments, de la fin du dix-neuvième siècle à la scène actuelle qui seront évoqués, à propos de l'enseignement du français aux étrangers et des formations de professeurs assurées en France. Ceci afin d'illustrer tant les configurations distinctes des circonstances où s'inscrivent ces formations que des figures d'acteurs à l'œuvre dans de telles configurations. Les dérivés nominaux en -tion ne manqueront pas à l'appel (didactisation, universitarisation, etc.), souvent peu esthétiques et euphoniques, mais ils permettront de désigner et de distinguer 
différents processus à l'œuvre dans les différentes phases de ce parcours d'un presque siècle.

\section{Un début d'institutionnalisation au cours des premières décennies de la Troisième République}

4 Avant de caractériser les transformations originales qui ont marqué les années 1960 puis les années 1980, il y a lieu de revenir sur l'état des lieux antérieur et sur les origines de cet état des lieux.

5 Jusqu'alors, deux types de lieux de formation ont existé en France pour qui entendait enseigner le français aux étrangers :

6 - D'une part, les formations assurées, dans un cadre universitaire, par l'École supérieure de préparation et de perfectionnement des professeurs de français à l'étranger (ESPPPFE) ${ }^{1}$, dont la fondation, due au linguiste Ferdinand Brunot (alors doyen de la faculté des lettres de la Sorbonne), remonte à 1920. Cette formation comporte des points communs avec celles proposées dans différents centres rattachés à des universités françaises, suisses et allemandes ${ }^{2}$, mais elle est caractérisée par sa spécialisation sur la préparation académique et professionnelle de futurs enseignants et d'enseignants déjà en exercice. Le cursus, au moment de la création, se déploie sur deux années et aboutit à un diplôme ${ }^{3}$. En 1945, la scolarité est portée à trois ans et, à partir de 1949, le diplôme vaut équivalence de licence de lettres modernes (Véronique 2010).

7 - D'autre part, des cours d'été s'étendant sur quelques semaines et axés en particulier sur l'oral (phonétique, conversation) et des éléments de civilisation. Ces cours d'été ont vu le jour à la toute fin du dix-neuvième siècle, dans la mouvance du mouvement de la Réforme, né en Allemagne, qui voit s'étendre en Europe les prosélytes de la phonétique, de la correction phonétique et de la méthode directe (Puren 1988; Galazzi 1995, 1997 ; Chevalier 1997, 2010 ; Chiss \& Coste 1995 ; Christ \& Coste 1993). L'Alliance française est active sur ce terrain et Ferdinand Brunot y est aussi impliqué très tôt (Chevalier 1997). Les programmes intéressent les enseignants étrangers, préfigurent en partie, dans les intitulés des enseignements, ce que seront ceux de l'EPPFE et peuvent mener à un diplôme spécifique de l'Alliance (Cortier 2005).

8 Ces formations à l'enseignement du français aux étrangers se présentent comme secondaires et marginales par rapport aux qualifications et diplomations relevant des cursus universitaires «classiques » menant à des diplômes d'études supérieures et, en France, aux concours de recrutement (agrégation et, plus tard, CAPES). Le degré de reconnaissance, de prise en compte ou d'équivalence de telle ou telle certification obtenue au titre de l'enseignement du français aux étrangers varie suivant les pays et les contextes. Dans bien des cas, les enseignants qui participent à des cours d'été ou suivent les enseignements de l'EPPFE ont déjà une formation académique et/ou sont déjà engagés dans l'enseignement aux étrangers. On dirait aujourd'hui que ce parcours complémentaire relève de la formation continue.

9 Si ces activités de formation s'adressent en particulier (mais non exclusivement) aux enseignants étrangers de français, elles se situent sur un arrière-plan - bien distinct de professionnalisation des enseignants de langues vivantes, professionnalisation dont quelques traits sont à souligner : 
10 - Dans la perspective de la préparation aux examens et aux concours de recrutement, des bulletins ou des revues ont vu le jour, qui apportent des connaissances et entrainent à des genres discursifs correspondant aux épreuves d'examens.

11 - Plus généralement, la pédagogie est à l'ordre du jour dans cette phase historique à la charnière $d u$ dix-neuvième et $d u$ vingtième siècles où la scolarisation gratuite et obligatoire se généralise ${ }^{4}$. Il s'agit certes d'éducation et non, spécifiquement, de langues modernes, mais ce climat pédagogique n'est pas sans affecter aussi la réflexion sur ces dernières.

12 - Les travaux relatifs au langage sont nombreux, s'agissant entre autres de sémantique et de lexicographie ou de phonétique. Une conception positiviste de la scientificité est prédominante, avec recours aux démarches expérimentales et aux outillages techniques.

13 - Les questions de méthodes et les débats parfois vifs auxquels elles se prêtent contribuent à la constitution d'un espace d'autonomisation et de professionnalisation spécifique des enseignants de langues modernes, eux-mêmes vite divisés entre partisans et adversaires de la méthodologie directe.

14 - Les échanges trouvent aussi à s'exprimer dans le cadre d'associations telles que l'Association des professeurs de langues vivantes (APLV), fondée en 1902, avec sa revue Les langues modernes, créée en 1903.

15 On a donc affaire à deux voies institutionnelles bien séparées, en coexistence mais à finalités et à publics différents, pour la formation des enseignants de langues. Celle, centrale, de la formation académique des enseignants d'allemand, d'anglais, etc. pour le système scolaire français. Celle, plus marginale, de la préparation ou du perfectionnement des professeurs de français à l'étranger. Le premier répond à une prise de conscience politique de l'importance croissante de la connaissance des langues européennes autres dans un moment de circulation et de compétition internationales accrues. Il trouve place dans des cursus universitaires menant à des titres nationaux et ouvrant à des concours de recrutement dans la fonction publique. Le second résulte d'une politique de présence et de diffusion de l'enseignement du français à l'étranger, chère à Brunot, historien de la langue française et du français « hors de France », mais tout aussi importante pour les pouvoirs publics avant et après la Première guerre mondiale (voir Chevalier 2010)5. Simplement, ce second dispositif, lieu de «préparation» et de "perfectionnement» pour l'enseignement du français aux étrangers a statut d'école, et ne pose pas de conditions académiques à l'admission des étrangers ${ }^{6}$.

16 Mais ces deux dispositifs institutionnels différents ne sont pas tout à fait étanches l'un par rapport à l'autre, du fait des facteurs contextuels transversaux relevés ci-dessus, du fait, notamment d'une certaine "pédagogisation» affectant les questions d'enseignement et, directement ou indirectement, les éléments de formation des enseignants.

17 Reste que, au regard de ce qui se met en place pour les professeurs de langues modernes dans le système scolaire national, la professionnalisation des enseignants de français aux étrangers au début du vingtième siècle est, en France, encore timide. Certes, on ne saurait négliger ce qui s'opère dans le cadre d'associations comme l'Alliance française ou la Mission laïque française, ni oublier le rôle des cours d'été ou, plus institutionnellement, à partir de 1920, les programmes de l'EPPFE. Mais il n'en 
reste pas moins que, dans les contenus de formation, et à en juger par les plans d'études de l'EPPFE naissante, l'accent porte essentiellement sur les composantes de la langue (vocabulaire du français, grammaire du français, orthographe du français, phonétique $\mathrm{du}$ français), sur la littérature française et sur des genres canoniques (composition française, explication de textes, exposé d'une question de grammaire). Les indications relatives aux techniques pédagogiques peuvent s'inscrire sous ces différentes têtes de chapitre, mais on voit bien que la spécificité du domaine de ce qui deviendra, des décennies plus tard, le FLE, n'est pas affirmée ${ }^{7}$.

Au bout du compte, dans cette période très active qui voit le passage du dix-neuvième au vingtième siècle, la formation en France des enseignants de français aux étrangers se caractérise par un début de professionnalisation surtout marquée par une première forme d'institutionnalisation, avec la création, en milieu universitaire, d'une école spécialisée accueillant des étudiants (ou déjà enseignants) français et étrangers et leur délivrant une reconnaissance de fin d'études, certification particulière et à la marge, sans équivalence alors avec d'autres diplômes du cursus des universités.

\section{L'impulsion des années 1960 : une spécialisation professionnelle reconnue}

19 C'est une nouvelle phase qui s'ouvre en France au début des années 1960, s'agissant de formation des enseignants de français aux étrangers. La configuration historique est connue. Après le retour aux affaires du général de Gaulle en 1958, une forte impulsion est donnée à l'action internationale de la France et, avec cette visée, à la diffusion du français dans le monde. Le ministère des Affaires étrangères est à la manœuvre et dispose de moyens importants, investis dans des plans quadriennaux. Mais le ministère de l'Éducation nationale est directement concerné, avec le détachement d'enseignants sur des postes et fonctions à l'étranger, ainsi que le ministère de la Coopération pour ce qui est des relations avec les anciennes colonies 8 .

Cette politique volontariste se traduit notamment, dès 1959, par la création d'organismes spécialisés, le Centre de recherche et d'étude pour la diffusion du français (CREDIF) qui succède au Centre d'étude du français élémentaire et est rattaché à l'École normale supérieure de Saint-Cloud, et le Bureau d'étude et de liaison pour l'enseignement $d u$ français dans le monde (BEL), qui sera rattaché à l'Institut pédagogique national et deviendra ensuite le BELC (Bureau pour l'enseignement de la langue et de la civilisation françaises à l'étranger), rattaché puis intégré au Centre international d'études pédagogiques de Sèvres (CIEP). Paul Rivenc, à la tête du CREDIF, et Guy Capelle, à la tête du BEL(C) vont initier des stages de formation et de spécialisation aux méthodologies nouvelles, destinés aux enseignants étrangers ou français enseignant à l'étranger. Les participants sélectionnés pour ces stages bénéficient pour la plupart de bourses couvrant les frais d'études et de séjour.

Des villes telles que Besançon, Montpellier, Aix-en-Provence deviendront des sortes de lieux de mémoire pour des cohortes d'enseignants passés par ces formations d'été de quatre à six semaines intensives ${ }^{9}$. Des stages de formateurs seront tôt organisés, afin notamment de démultiplier dans des pays étrangers des opérations similaires de formation continue. Et seront aussi mis en place à la fin des années 1960, des stages d'un an, dits "stages longs", au BELC et au CREDIF, ouverts à des petits groupes d'enseignants français ou étrangers appelés à remplir des fonctions importantes de 
conseil et de formation dans des postes culturels français à l'étranger ou des institutions étrangères.

Il n'y a pas lieu de s'étendre ici sur le détail de ces évolutions ni sur le contenu précis des formations ${ }^{10}$. Il s'agit plutôt de pointer des traits qui rapprochent ou différencient ce moment historique de celui évoqué précédemment.

23 - Dans un cas comme dans l'autre, le contexte politique est propice à des actions en faveur de l'enseignement et de la diffusion du français à l'étranger, mais cette volonté institutionnelle se traduit par la mise en œuvre de moyens beaucoup plus importants dans les années 60 .

24 - Au tournant entre dix-neuvième et vingtième siècles, les pouvoirs publics apportent un certain appui à des associations comme l'Alliance française et la Mission laïque française, voire aux initiatives du doyen Brunot lors de la création de l'EPPFE. Mais en 1960, avec le BEL et le CREDIF, ce sont des centres, certes marginaux, mais dépendant de la sphère publique et en «prise directe » avec les ministères concernés qui sont créés.

25 - Cela se manifeste aussi pour ces instruments particuliers que sont les revues ou bulletins. La Mission laïque française publie une Revue de l'enseignement français colonial, dont le premier numéro paraît en 1904 et qui devient en 1907 le Bulletin de la Mission laïque française (Thévenin 2002). Créée en 1883, l'Alliance française se dote très tôt (1884) d'un Bulletin de l'Alliance française, puis, à partir de 1920, d'une Revue de l'Alliance française et, en 1948, d'un nouveau bulletin, L'enseignement du français aux étrangers, rédigé par des professeurs de l'Alliance (Bruézière 1983). Ce dernier outil, apparu après la Deuxième Guerre mondiale, est le seul qui ait vraiment eu une fonction pédagogique de soutien aux enseignants des alliances françaises dans une perspective de formation continue pratique. Mais c'est avec la création, en 1961, de la revue Le français dans le monde, dont l'initiateur et premier rédacteur en chef est André Reboullet, qu'un instrument destiné aux enseignants de ce qu'on commence alors à nommer « français langue étrangère » est lancé ${ }^{11}$ (Reboullet 1984).

26 - Dans un cas comme dans l'autre, c'est aussi un environnement scientifique et technologique porteur que l'on observe (modèles linguistiques nouveaux, conceptions de l'apprentissage, aides techniques sonores et visuelles), mais ces dimensions sont plus fortement revendiquées dans les années 60 , au titre de la linguistique appliquée, du structuralisme ou du courant structuro-global que dans la période précédente.

27 - Mais la différence majeure consiste en ce que l'objet lui-même, le français, est désormais considéré sous un autre angle, ce que manifeste la notion même de « français langue étrangère ». L'élaboration du français fondamental au cours des années 1950 et les violentes attaques auxquelles elle a été soumise ont été le signe que les représentations dominantes du français étaient d'une certaine manière ébranlées et en train d'évoluer (Coste 2006). C'est autour de la question alors perçue comme provocatrice, "Quel français enseigner?", que s'ordonnent de nouvelles approches de la langue et de nouvelles conceptions de l'apprentissage, cependant que de nouveaux supports et de nouvelles démarches d'enseignement sont proposées, tout ceci impliquant à tout le moins un « recyclage » et une formation adéquate des enseignants.

28 - On attend désormais des déjà ou futurs enseignants de français à l'étranger qu'ils deviennent ainsi des spécialistes du français langue étrangère. Méthodes nouvelles (audio-orale, SGAV), techniques nouvelles (audiovisuelles) objectivées dans des 
manuels et supports nouveaux (Voix et Images de France, Bonjour Line, La France en direct) sont la marque d'une spécialisation qui passe par une "méthodologisation " et une manuélisation » fortement affirmées, adossées à un argumentaire théorique présenté comme garantissant la cohérence de l'ensemble ${ }^{12}$.

- La formation des enseignants reste un complément de formation, mais, à la différence de ce qui se passait au début du vingtième siècle, cette formation s'inscrit dans une dynamique de convergence entre des instances faisant œuvre commune. Ainsi, le CREDIF assure des stages de spécialisation à l'enseignement audiovisuel autour de l'utilisation de la méthode Voix et Images de France et délivre des diplômes d'aptitude à l'issue d'épreuves théoriques et pratiques. L'obtention de ce diplôme conditionne la commande commerciale de ladite méthode chez l'éditeur Didier et, pour nombre de centres d'enseignement étrangers ou relevant d'un dispositif français à l'étranger (alliances, centres culturels, etc.), c'est le ministère des Affaires étrangères qui finance, en tout ou en partie, l'acquisition de ce matériel d'enseignement, ayant par ailleurs souvent pris en charge les frais de stage par une bourse d'études.

C'est dans ce contexte général que s'élabore peu à peu, entre linguistique appliquée et méthodologie de l'enseignement des langues, une didactisation originale du français langue étrangère, intégrant étroitement appareillage théorique et démarches pratiques $^{13}$. Et il est permis de penser que les stages de formation d'enseignants, de par la portée argumentative et démonstrative qu'ils ont à assurer, ont fortement contribué à la mise en œuvre de cette didactisation bien plus qu'ils n'en auraient été une résultante.

\section{Le coup de force des années 1980 : l'universitarisation d'une discipline}

31 Troisième moment et troisième configuration particulière pour le parcours ici proposé. Le retour au pouvoir du général de Gaulle avait été marqué par un renforcement et une réorientation notables de la politique linguistique extérieure de la France; le début du premier septennat de François Mitterrand, élu président de la République en 1981, voit une relance des actions relatives à la diffusion du français à l'étranger, à l'organisation des cours de français langue étrangère en France et à la reconnaissance universitaire du domaine.

Le nom de Louis Porcher, professeur des universités et alors conseiller auprès du directeur des relations internationales au ministère de l'Éducation nationale, est étroitement associé à ces évolutions décisives. Rapporteur d'une commission mise en place par le ministre et présidée par le recteur Jean Auba, Louis Porcher est la cheville ouvrière de propositions qui vont conduire à deux innovations majeures (Porcher 1986 ; voir aussi Les Cahiers de l'Asdifle 1, 1988). D'une part sont créés deux diplômes nationaux attestant d'une maîtrise du français, le diplôme élémentaire de langue française (DELF) et le diplôme approfondi de langue française (DALF). D'autre part et surtout est instaurée une filière universitaire spécifique pour le français langue étrangère (FLE), comportant une mention FLE adjointe à certaines licences et une maitrise FLE de plein exercice, dont les contenus sont définis par une maquette nationale. Le tout pouvant éventuellement se poursuivre, au troisième cycle, par un diplôme d'études approfondies et un doctorat ou un diplôme d'études supérieures 
spécialisées à orientation plus tournée vers les métiers (formation, édition, communication) liés au français langue étrangère, à l'enseignement des langues et aux politiques linguistiques.

Il y a là une sorte de coup de force avec la mise en place d'un cursus de formation universitaire reconnu donnant lieu à des titres, à l'égal d'autres disciplines. Longtemps à la marge du système éducatif, la formation d'enseignants de français aux étrangers apparaît dès lors comme intégrée pleinement à l'appareil de l'enseignement supérieur et pouvant déboucher, à différents niveaux d'études, sur des emplois dans le domaine.

L'intérêt pour cette nouvelle filière s'avérera être vif et sera à l'origine de création de postes universitaires (maitre de conférences, professeurs), à l'intérieur notamment des départements ou unités de sciences du langage. Même si elles lui sont contestées par d'autres disciplines plus anciennement établies, le FLE a reçu ses lettres de noblesse et trouvé une place à l'intérieur des formations académiques initiales.

Si ce coup réussit, dans la mouvance d'un changement politique, il ne devrait pas faire oublier que d'autres tentatives l'ont précédé mais n'avaient abouti que partiellement ou temporairement. Et cela, comme le rappelle Véronique (2010), dans le cadre et à l'initiative de l'ESPPPFE. L'École a connu diverses évolutions dans le cadre universitaire après 1945 : passage de la scolarité de deux à trois ans et équivalence de licence pour le diplôme de professeur de français à l'étranger, sanctionnant la fin des études; préparation à la mention étranger du CAPES de lettres modernes (jusqu'à la fin des années 1970). L'ESPPPFE a changé d'intitulé en 1962, devenant alors Institut des professeurs de français à l'étranger (IPFE). Dans la suite immédiate de la loi d'orientation de l'enseignement supérieur de novembre 1968 (configuration nouvelle, après les « événements » de mai 68), nouveau changement : en 1969, l'institut est érigé en Unité d'enseignement et de recherche avec la désignation « Études françaises pour l'étranger » (UER-EFPE) à l'intérieur de l'université Sorbonne Nouvelle - Paris 3. C'est dans cette structure que Robert Galisson, docteur d'État en $1976^{14}$ (thèse dirigée par Bernard Quemada) va lui-même inscrire et diriger, jusqu'à la fin de sa carrière, de très nombreuses thèses en didactique / didactologie des langues, rejoint en cela par Louis Porcher, qui, à partir de $1983^{15}$, a une chaire dans cette même UER. Il y a donc une forte continuité entre la création de Ferdinand Brunot en 1920 et ce qui est devenu aujourd'hui, près d'un siècle plus tard, le département de didactique du français langue étrangère ${ }^{16}$.

36 Ce en quoi la mise en place de la filière FLE au milieu des années 1980 constitue une rupture avec ce qui a précédé, c'est évidemment son caractère national. De très nombreuses universités vont d'autant plus s'engager dans cette voie offerte que l'obtention de la maîtrise FLE devient un quasi sinequanone pour les (futurs) enseignants qui souhaitent partir enseigner le français à l'étranger. Ce trait n'est pas sans rappeler le caractère de prérequis qu'avait le diplôme du CREDIF, dans les années 1960, pour l'utilisation des nouvelles méthodes.

37 La différence avec ce qui a été dit plus haut de ces années 1960 tient à ce que la création et la forte extension de la filière FLE dans les universités associe la formation des enseignants à une disciplinarisation et à une curricularisation universitaires, mais ne comporte pas les dimensions de "manuélisation» et de "méthodologisation » qui caractérisaient les stages de formation du CREDIF et du BELC. Dès lors que les formations au FLE s'inscrivent dans une logique de transmission / construction de connaissances autour et à propos de l'enseignement et de l'apprentissage du FLE, on 
n'est plus dans un projet visant à nouer des rapports de cohérence étroite entre des argumentaires théoriques et des démarches pédagogiques.

De manière qui peut passer pour paradoxale, c'est l'autre pilier de la construction institutionnelle réalisée dans les années 1980 qui va jouer un rôle de régulation pédagogique et constituer une modalité informelle de la formation pratique des enseignants de FLE. En effet, tout comme la filière FLE, les diplômes nationaux DELF et DALF trouvent très vite un large accueil dans les cursus d'apprentissage du FLE et le marché de l'édition s'emploie à produire des manuels qui prennent en compte la visée et l'atteinte de ces objectifs de certification. Les manuels deviennent pour partie - et en tout cas beaucoup plus qu'auparavant - des outils de préparation à des épreuves d'évaluation validant un niveau de compétence linguistique et apportant une reconnaissance sur des marchés où ces compétences certifiées sont exigées ou appréciées. C'est en quelque sorte par ricochet que ce type de manuélisation en vient à jouer un rôle dans la formation au continu des enseignants en service. Il ne s'agit plus, comme dans les années 1960, d'un dispositif cohésif et cohérent où la méthodologie apparaissait comme le centre de gravité de l'ensemble et où la manuélisation était partie intégrante de la formation des enseignants de FLE. On assiste plutôt à une répartition tacite des rôles et à une complémentarité officieuse entre formation universitaire certifiée des enseignants et apports pédagogiques pratiques articulés à un outil de certification des apprenants. Dans les meilleurs des cas et du fait que les contenus de la filière FLE, d'un côté, les modules des certifications DELF / DALF de l'autre ont été pensés et définis dans la dynamique d'un même projet et selon des inspirations similaires, cette complémentarité officieuse peut s'avérer fonctionnelle.

\section{La scène actuelle : internationalisation, diversification et fragmentation?}

Quitte à renoncer à ce minimum de recul qu'autorise une mise en perspective diachronique, la considération de la situation actuelle en France ouvre des éléments nouveaux à l'analyse. Et d'abord le fait que la scène française pour la formation des enseignants de français langue étrangère se trouve marquée par, d'une part, des traits extrêmement spécifiques et d'autre part des influences internationales, non limitées d'ailleurs au contexte européen. Au demeurant, les spécificités nationales ne sont que partiellement séparables de phénomènes qui interviennent à une plus grande échelle et affectent de manière particulière la situation du français.

On relèvera ici :

- une réflexion européenne sur la formation des enseignants de langues et sur les instruments ou référentiels convenant à cette formation. On notera par exemple des mentions fréquentes du rapport remis par Michael Kelly à la Commission européenne (Kelly et al. 2004), qui propose un cadre de référence européen pour la formation des enseignants de langues étrangères. Au titre du Conseil de l'Europe, le Centre européen pour les langues vivantes de Graz a accompagné un certain nombre de projets confiés à des équipes internationales et proposant aussi des référentiels ou des portfolios pour la formation initiale ou continue des enseignants de langues (voir notamment les projets PEPELF et CARAP $^{17}$ ). Ne manquent pas par ailleurs les publications collectives sur ces 
questions (par exemple : Causa 2012 ; Causa, Galligani, Vlad 2014 ou encore Les Cahiers de l'Asdifle 1, 1988 et 11, 1999);

- une incidence sur cette réflexion et ces propositions européennes des courants qui, depuis une vingtaine d'années, ont souligné l'importance du plurilinguisme et de la prise en compte et de la promotion de la diversité linguistique et culturelle. Et ce au détriment, selon certains auteurs (voir Maurer 2011, Chiss 2013), des contenus de formation touchant aux caractéristiques linguistiques de la langue à enseigner et de son apprentissage ;

- sur la scène française, une double tendance, qui n'est paradoxale qu'en apparence, à la fusion transversale et à la fragmentation ciblée. Fusion transversale dès lors que la diversité linguistique et culturelle réduit les cloisonnements entre les langues singulières et les publics auparavant très séparés par l'analyse didactique : il en va ainsi de la notion de FLES, qui neutralise en partie la séparation longtemps tenue entre français langue étrangère (FLE) et français langue seconde (FLS) et va dans le sens d'une ouverture plus marquée de la formation des enseignants. Mais fragmentation ciblée aussi, dès lors que, complémentairement à la fusion, les distinctions s'affirment pour d'autres "segments" des publics potentiels ou actuels à privilégier: français sur objectifs spécifiques (FOS), français sur objectifs universitaires (FOU), français langue de scolarisation (FLSCO), voire le très débattu FLI (français langue d'intégration).

Cette double tendance est évidemment de nature à affecter en France la formation des enseignants de français langue étrangère. Ce d'autant plus que des phénomènes propres au français, sur le plan national ou au niveau international, influent sur le paysage de la formation.

45 - La mastérisation des formations initiales (suppression du niveau maîtrise après quatre ans d'études universitaires et la mise en place de mastères après cinq ans), importante réforme des études, a entrainé la disparition des maîtrises de français langue étrangère, alors que cette sorte d'étalon des formations avait géré et calibré les formations pendant une trentaine d'années et structuré de fait la scène nationale. Dès lors que cette maquette commune de référence s'efface au profit de mastères qui tendent à se différencier tant soit peu les uns des autres dans l'espace universitaire national, la formation des enseignants répond désormais en partie à des stratégies locales qui, sans tenir certes de la marchandisation, intègrent une dimension concurrentielle et ont à résoudre chacune la délicate question de l'équilibre à trouver entre tronc commun des formations et degré de spécialisation sur des «cibles » plus spécifiques, en particulier pour ce qui est des mastères "pro" (professionnalisants) par rapport aux mastères "recherche» inscrits dans la logique des études doctorales ${ }^{18}$. En outre, le développement universitaire du complexe FLE/didactique des langues, son affirmation dans la recherche et les relations qui s'opèrent légitimement entre la diversification des recherches et les contenus de formation peuvent induire un décrochage relatif entre ces derniers et ce qui touche aux pratiques ordinaires des enseignants ${ }^{19}$.

- L'impact des niveaux de compétence définis par le Cadre européen commun de référence pour les langues (CECR) sur les reconnaissances institutionnelles de maittrise d'une langue (examens, certifications) marque en profondeur la conception des manuels d'enseignement du français. La généralisation de validations aux niveaux A2, B2, etc. (y compris dans les évolutions qu'elle entraine pour le DELF et ses dérivés actuels) a des effets en retour sur ce qui est attendu des enseignants, alors même que ces derniers ont de plus en plus accès à une abondance de ressources pédagogiques en ligne, peuvent se 
sensibiliser aux approches plurielles (intercompréhension, éveil aux langues, réflexion interculturelle, didactique intégrée des langues, enseignement bi-/plurilingue), sont encouragés à la prise en compte des répertoires langagiers des apprenants. Des tensions naissent entre, d'une part, la tendance à une homogénéisation de démarches qui seraient toutes orientées vers un même mode d'évaluation et, de l'autre, l'incitation à localiser et diversifier les modalités d'enseignement.

47 - Quant à la sphère internationale, une forme d'image miroir se présente, due à deux facteurs non directement liés, mais qui à plus d'un égard se complètent. D'un côté, dans bien des pays (dont nombre d'européens), l'enseignement du français, en milieu scolaire et ailleurs, paraît plus en régression qu'en progression et il n'est plus impératif de former de nouveaux enseignants: la demande de formation se raréfie et/ou se focalise sur des situations et des secteurs particuliers. D'un autre côté, les enjeux et les dynamiques de la francophonie pèsent aussi dans le sens d'une contextualisation accrue des aides à la formation des enseignants ou dans celui de la recherche de dispositifs passant par la distance, les programmes en ligne, des MOOCs de la formation.

Cette dernière évolution est particulièrement significative. Elle correspond à une diversification des supports et des modalités de ces formations, ainsi qu'à une certaine multiplication des opérateurs, au fur et à mesure que les « métiers du FLE » (Les Cahiers de l'Asdifle 16, 2004) se différencient et se distinguent (voire s'éloignent de plus en plus) de la figure classique et canonique de l'enseignant face à une classe d'élèves, d'étudiants ou d'adultes en formation ${ }^{20}$.

C'est donc un panorama contrasté et de lecture assez difficile (pas simplement pour des raisons de proximité et de "nez sur l'événement ») que l'on découvre et dans lequel on est plongé aujourd'hui en France; et force est de constater que, pour ce qui est du français langue étrangère, ni la création en 2013 des écoles supérieures du professorat et de l'éducation (ESPE), ni l'instauration d'une option FLE au Capes de lettres modernes ne semblent de nature, pour le moment du moins, à modifier et clarifier la situation $^{21}$. Il est permis de se demander si on assiste, pour ce qui relève de la France, à une complexification et à des ajustements de plus en plus fins des formations ou bien à une dilution généralisée des programmes après la phase d'universitarisation régulée engagée au cours des années 1980.

\section{BIBLIOGRAPHIE}

BERTHET, Michel (2010). « De l'IPFE à l'UFR de didactique du français langue étrangère. Enjeux disciplinaires et institutionnels (1960-2000) », Documents pour l'histoire du français langue étrangère ou seconde, 44, 111-128.

BRUÉZIÈRE, Maurice (1983). L'Alliance française : histoire d'une institution, l883-1983. Paris : Hachette. BuIsson, Ferdinand (dir.) (1887). Dictionnaire de pédagogie et d'instruction primaire (tome 1 et tome 2). Paris : Hachette (accessible en ligne sur Gallica). 
BUISSON, Ferdinand (dir.) (1911). Nouveau dictionnaire de pédagogie et d'instruction primaire. Paris : Hachette (accessible en ligne sur le site de l'IFÉ).

CAUSA, Mariella (dir.) (2012). Formation initiale et profils d'enseignants de langues. Enjeux et questionnements. De Boeck Supérieur.

CAUSA, Mariella, GALLIGANI, Stéphanie \& VLAD Monica (dir.) (2014). Formation et pratiques enseignantes en contextes pluriels. Paris : Riveneuve Éditions.

CHEVALIER, Jean-Claude (1997). «Phonétique expérimentale et Cours pour étrangers en France à la fin du $19^{\mathrm{e}}$ siècle ", Documents pour l'histoire du français langue étrangère ou seconde, 19, 158-165.

CHEVALIER, Jean-Claude (2010). « Ferdinand Brunot et les débuts de l'École de préparation des professeurs de français à l'étranger », Documents pour l'histoire du français langue étrangère ou seconde, 44, 15-28.

CHISS, Jean-Louis \& COSTE, Daniel (dir.) (1995). « Théories du langage et enseignement / apprentissage des langues (fin du XIX ${ }^{\mathrm{e}}$ siècle - début du XX $\mathrm{X}^{\mathrm{e}}$ siècle », Histoire Épistémologie Langage, $\mathrm{XVII} / \mathrm{I}$.

CHRIST, Herbert \& COSTE, Daniel (dir.) (1993). « Pour et contre la méthode directe. Historique du mouvement de réforme de l'enseignement des langues de 1880 à 1914 ", Études de Linguistique Appliquée (ELA), 90.

CHISS, Jean-Louis (2013). « La didactique du français et des langues à l'épreuve de la pluralité linguistique et culturelle ». In Jean-Claude Beacco (dir.), Éthique et politique en didactique des langues. Paris : Didier, 99-112.

COSTE, Daniel (dir.) (1984). Aspects d'une politique de diffusion du français langue étrangère depuis 1945. Matériaux pour une histoire. Paris : Hatier.

COSTE, Daniel (2006). « Français élémentaire, débats publics et représentations de la langue », Documents pour l'histoire du français langue étrangère ou seconde, 36, 13-33.

COSTE, Daniel (2010). « Note sur la création de l'École de préparation des professeurs de français à l'étranger », Documents pour l'histoire du français langue étrangère ou seconde, 44, 29-40.

CUQ, Jean-Pierre \& KAHN, Gisèle (dir.) (1997). « L'apport des centres de français langue étrangère à la didactique des langues ", Documents pour l'histoire du français langue étrangère ou seconde, 20.

DABÈNE, Michel (1986) «Ralentir travaux ! Sur quelques évidences en didactique du français langue étrangère », Études de Linguistique Appliquée (ELA), 64, 31-38.

GALAZZI, Enrica (1995). «Phonétique/Université/Enseignement des langues à la fin du XIX ${ }^{\mathrm{e}}$ siècle ", Histoire Épistémologie Langage, XVII/I, 95-114.

GALAZZI, Enrica (1997). « Théodore Rosset : une méthode originale pour l'enseignement de la prononciation française à l'Université de Grenoble dès 1904 ", Documents pour l'histoire du français langue étrangère ou seconde, 20, 37-72.

GALISSON, Robert (1977). «S.O.S... Didactique des langues étrangères en danger... Intendance ne suit plus... S.O.S... », Études de Linguistique Appliquée (ELA), 27, 78-98.

GALISSON, Robert \& COSTE, Daniel (dir.) (1976). Dictionnaire de didactique des langues. Paris : Hachette.

KELLY, Michael, GRENFELL, Michael, ALLAN, Rebecca, KRIZA, Christine \& McEVOY, William (2004). European Profile for Language Teacher Education. A Frame of Reference. A Report to the European Commission. 
Les Cahiers de l'Asdifle, 1 (1988). « Formation en didactique des langues étrangères ».

Les Cahiers de l'Asdifle, 11 (1999). « De nouvelles voies pour la formation ».

Les Cahiers de l'Asdifle, 16 (2004). « Les métiers du FLE ».

MAURER, Bruno (2011). Enseignement des langues et construction européenne. Le plurilinguisme, nouvelle idéologie dominante. Paris : Éditions des archives contemporaines.

PORCHER, Louis (1986). « Priorités institutionnelles », Études de Linguistique Appliquée (ELA), 64, $75-92$.

PUREN, Christian (1998) [1988]). Histoire des méthodologies de l'enseignement des langues. Paris : Nathan CLE International.

REBOULLET, André (1984). « Presse pédagogique, enseignants et enseignement du français. 1950-1970 ». In Daniel Coste (dir.), Aspects d'une politique de diffusion du français langue étrangère depuis 1945. Matériaux pour une histoire. Paris : Hatier, 106-119.

SPAËTH, Valérie (2010a). « Les institutions de diffusion du français et l'EPPFE en 1920 : l'universalité du français en question? ", Documents pour l'histoire du français langue étrangère ou seconde, 44, 41-54.

SPAËTH, Valérie (2010b). « Mondialisation du français dans la seconde partie du XIX ${ }^{\mathrm{e}}$ siècle : l'Alliance Israélite Universelle et l'Alliance Française », Langue française, 167, 49-72.

THÉVENIN, André (2002). La Mission laïque française à travers son histoire. 1902-2002. Paris : Mission laïque française.

VÉRONIQUE, Georges Daniel (2010). « De l'ESPPPFE à l'UER EFPE : l'émergence d'un acteur universitaire de la didactique du FLE (1945-1980) », Documents pour l'histoire du français langue étrangère ou seconde, 44, 89-102.

\section{NOTES}

1. La désignation de cette institution, aujourd'hui département de didactique du français langue étrangère de l'université Sorbonne Nouvelle - Paris 3, a connu de multiples fluctuations. À sa création, en 1920, elle est nommée École de préparation des professeurs de français à l'étranger (EPPFE). Le premier projet présenté par Brunot prévoyait de l'intituler École normale des professeurs de français à l'étranger. Sur l'histoire de cet important lieu de formation, voir le $\mathrm{n}^{\circ}$ 44 de Documents pour l'histoire du français langue étrangère ou seconde (juin 2010) et en particulier les articles de Berthet, Coste, Spaëth, Véronique.

2. Voir le $n^{\circ} 20$ (Cuq \& Kahn 1997) de Documents pour l'histoire du français langue étrangère ou seconde portant sur «L'apport des centres de français langue étrangère à la didactique des langues ".

3. Sur les programmes des cours et les épreuves d'examens au moment de la création, voir coste 2010.

4. Mouvement marqué par le Dictionnaire de pédagogie de Ferdinand Buisson, qui connaît deux éditions (1887 et 1911 ; cette seconde édition est intitulée Nouveau dictionnaire de pédagogie et est profondément actualisée et remaniée, suite à la réforme de 1902. Voir à ce propos, sur le site de l'Institut français de l'éducation (IFÉ) :<http://www.inrp.fr/edition-electronique/lodel/ dictionnaire-ferdinand-buisson/>

5. La période avant et après la Première Guerre mondiale est celle qui voit la création d'Instituts culturels et de lycées français dans de nombreux pays étrangers, en particulier européens. 
6. La référence, comme l'indique le premier titre envisagé par Brunot (cf. ci-dessus note 1), est celle des écoles normales d'instituteurs et en quelque manière celle de l'enseignement primaire. Il est significatif que, dès sa création comme association en 1902, la Mission laïque française, qui intervient alors aussi dans les colonies ait ouvert une " école normale d'enseignement colonial », l'École Jules Ferry. Mais dès avant, l'Alliance israélite universelle, créée en 1860, avait ouvert en 1865 une École normale israélite orientale (ENIO), qui existe toujours, pour la formation des enseignants. Pour une comparaison entre l'Alliance israélite universelle et l'Alliance française, voir Spaëth 2010b.

7. On peut estimer que la circulation méthodologique est plus visible, s'agissant surtout de la méthode directe (recommandée ou contestée) entre l'enseignement primaire du français et en français en contexte de langue régionale, l'enseignement dans les colonies et l'enseignement des langues modernes dans le secondaire.

8. Il faut rappeler aussi que, dans les années 1960 et 1970, un très grand nombre d'enseignants détachés et de jeunes au titre du service de coopération seront engagés dans une politique d'aide au développement des systèmes éducatifs des pays nouvellement indépendants, surtout au Maghreb et dans les pays de la zone francophone d'Afrique subsaharienne. Cette coopération dite de substitution fera naître chez certains une vocation pour l'enseignement aux étrangers et pour le français langue étrangère ou seconde, mais la formation spécialisée de ces coopérants sera loin d'être assurée, si ce n'est pour ceux, une minorité parmi les enseignants titulaires, qui passeront par les stages d'été du BELC ou du CREDIF ou bénéficieront de structures locales, comme au Maroc avec le Bureau d'études et de recherches pédagogiques, créé auprès de la Mission culturelle française.

9. De nombreuses autres villes accueilleront les stages du BELC (jusqu'à aujourd'hui) et ceux du CREDIF (jusqu'en 1996) et d'autres centres de langues organiseront des stages similaires dès le milieu des années 1960 (par exemple, Vichy et Royan), cependant qu'à Besançon, le Centre de linguistique appliquée (CLA), fondé par Bernard Quemada, organise ses propres stages d'été.

10. On pourra se reporter à l'ouvrage Aspects d'une politique de diffusion du français langue étrangère depuis 1945. Matériaux pour une histoire (Coste, dir., 1984) qui regroupe les témoignages de nombre des protagonistes de ces évolutions.

11. Deux éditeurs, Hachette et Larousse, s'associent pour cette entreprise, mais grâce aux commandes importantes du ministère des Affaires étrangères et à la mise à disposition de deux postes d'enseignants par le ministère de l'Éducation nationale.

12. Cette insistance sur la nouveauté et une certaine scientificité se double d'un positionnement critique à l'encontre des méthodes vite qualifiées de "traditionnelles». Par contrecoup, des formations comme celles assurées par l'ESPPPFE ou par l'Alliance française en viennent à être vues comme ringardes. Ce qui ne rend pas justice aux évolutions qu'elles connaissent alors, en particulier l'ESPPPFE (voir ci-après).

13. À l'articulation entre affirmation théorisante d'un secteur nouveau et contribution indirecte à la formation des enseignants, les collections créées dans les années 1970 et 1980 présentent des positionnements distincts et significatifs. La collection F, créée par André Reboullet chez Hachette se donne une orientation plus pédagogique que la collection Didactique des langues étrangères (DLE) que Robert Galisson, avec une orientation méthodologique, dirige chez CLE International ou encore que Langues et apprentissage des langues (LAL), qu'animent Henri Besse et Daniel Coste, chez Hatier puis Didier au titre du CREDIF et qui s'ouvre à des apports théoriques de disciplines connexes et de courants internationaux.

14. Année de publication du Dictionnaire de didactique des langues (Galisson \& Coste, 1976), qui marque une étape dans la constitution du domaine de la didactique du FLE et des langues. André Reboullet, rédacteur en chef $d u$ français dans le monde et initiateur du projet de dictionnaire, souhaitait que ce dernier soit aussi un instrument de formation pour les enseignants. La revue Le français dans le monde a connu elle-même une évolution comparable dans les années 1970-1980 : 
pensée à l'origine comme un instrument servant la professionnalisation des enseignants, elle devient aussi (sous l'influence des contributeurs du BELC et du CREDIF et surtout à travers ses numéros spéciaux) un cheval de Troie de la méthodologisation et de la didactisation du domaine $\mathrm{du}$ français langue étrangère.

15. Année où se préparent les textes de création de la filière FLE.

16. À raisonner en termes de rivalités institutionnelles dans un même champ, on pourrait dire que, si les années 1960 et 1970 avaient été marquées par la dynamique dominante des centres BELC et CREDIF, des lieux universitaires comme Paris 3 vont s'affirmer à partir des années 1980 en position forte et centrale. Ceci n'est pas sans lien avec les changements affectant ensuite les centres historiques: le BELC est pleinement intégré au CIEP en 1987 et, dans un contexte d'évolutions plus complexes, le CREDIF est dissous par le directeur de l'ENS de Fontenay-SaintCloud en 1996.

17. <http://www.ecml.at/Resources/ECMLPublications/tabid/277/Themes/tee/ PublicationID/-1/language/fr-FR/Default.aspx> sur le site du CELV.

18.

Voir le Répertoire des Masters en FLE, établi et tenu à jour par le CIEP (Centre international d'études pédagogiques) de Sèvres, en liaison avec l'Asdifle (Association de didactique du FLE) et le Buffle (Bureau des filières de FLE) : <http://www.ciep.fr/ressources/repertoire-masters-fle>

19.

Ce constat d'un décalage perçu entre contenus de formation universitaire et réalités des besoins du «terrain » est fréquent et non propre au domaine considéré, mais il est dressé dès les années 1980, y compris par des didacticiens universitaires (par exemple : Galisson 1977, Dabène 1986).

20.

On notera ainsi que, dans le cadre du CIEP, opérateur du ministère de l'Éducation nationale, ce qui reste de l'ancien BELC, à savoir avant tout des stages de courte durée pour enseignants, en hiver et en été, est désormais dénommé " Université BELC - Les métiers du français dans le monde ». L'Institut français, opérateur du ministère des Affaires étrangères, propose, lui, en direction des enseignants de français sur objectifs spécifiques, Numérifos, une banque de ressources comportant en ligne des dossiers pédagogiques, et IFOS, une plateforme de formation à distance pour les professeurs de français professionnel.

21.

Voir toutefois le billet d'Isabelle Gruca du 25 novembre 2014 sur le site de l'AFEF (Association française des enseignants de français): <http://www.afef.org/blog/post-les-enjeux-de-lacreation-d-une-option-fle-au-capes-de-lettres-par-isabelle-gruca-p1163-c48.html>

\section{RÉSUMÉS}

L'article distingue quatre moments majeurs dans l'évolution de la formation en France des enseignants de français langue étrangère : (1) une première institutionnalisation au début du XXe siècle ; (2) une spécialisation et une didactisation dans les années 1960 ; (3) une universitarisation et une disciplinarisation à partir des années 1980 ; (4) une diversification et une fragmentation relative en ce début du XXIe siècle. Ces différentes phases tendent à s'inscrire dans des configurations politiques, économiques, scientifiques où des évolutions importantes interviennent. 
Four principal moments are characterised in the evolution in France of teacher development for French as a foreign language from the end of the 19th century to the present time : (i) a first institutionalisation at the beginning of the $20 \mathrm{~h}$ century ; (ii) a specialisation and a "didactisation" in the 1960's ; (iii) a universitarisation process and an academic recognition from the 1990's on ; (iv) at present, forms of fragmentation and diversification. These different phases seem to occur in contexts where political, economic and scientific changes take place.

\section{INDEX}

Mots-clés : formation des enseignants, français langue étrangère, France, XXe siècle.

Keywords : teacher education, French as a foreign language, France, 20th century.

\section{AUTEUR}

\section{DANIEL COSTE}

ENS de Lyon 